Wellbeing at Work: Four Perspectives on What User Experiences with Artifacts May Contribute

Hertzum, Morten

Published in:

Beyond Interactions - Revised Selected Papers from INTERACT2019 IFIP TC 13 Workshops

DOI:

10.1007/978-3-030-46540-7_2

Publication date:

2020

Citation for published version (APA):

Hertzum, M. (2020). Wellbeing at Work: Four Perspectives on What User Experiences with Artifacts May

Contribute. In Beyond Interactions - Revised Selected Papers from INTERACT2019 IFIP TC 13 Workshops (Vol. LNCS 11930, pp. 19-25). Springer. Lecture Notes in Computer Science https://doi.org/10.1007/978-3-03046540-7_2 
In J.A. Nocera, A. Parmaxi, M. Winckler, F. Loizides, C. Ardito, G. Bhutkar, and P. Dannenmann (eds.) (2020), Beyond Interactions - Revised Selected Papers from INTERACT2019 IFIP TC 13 Workshops. LNCS 11930, pp. 19-25. Springer Nature, Cham.

\title{
Wellbeing at Work: Four Perspectives on What User Experiences with Artifacts May contribute
}

\author{
Morten Hertzum ${ }^{[0000-0003-0019-8531]}$ \\ University of Copenhagen, Copenhagen, Denmark \\ hertzumehum. ku.dk
}

\begin{abstract}
Most work involves the use of artifacts; thus, user experience (UX) is a factor in how most employees experience their work. This study revisits the tool, media, dialogue-partner, and system perspectives on artifact use to explore how UX may contribute to wellbeing at work. It is found that artifacts foster positive UX when they lend the user expressive power (tool), are transparent (media) or perceptive (dialogue partner). They foster negative UX when they break the user's task focus or make the user a mere system component. These findings are discussed and refined by elaborating the classic concepts of ready to hand and present at hand.
\end{abstract}

Keywords: Perspectives on artifact use, User experience, Wellbeing, Work.

\section{Introduction}

Wellbeing at work $[2,4]$ is a major concern for employees as well as organizations because it is central to employees' mental and physical health and because it influences their productivity. Fisher [6] conceptualizes wellbeing at work as consisting of hedonic wellbeing (e.g., job satisfaction and positive affect), eudaimonic wellbeing (e.g., engagement, meaning, and intrinsic motivation), and social wellbeing (e.g., quality connections, satisfaction with coworkers, and social support). This conceptualization makes it apparent that a vast array of concrete organizational circumstances enter into shaping wellbeing at work. One of them is the employees' use of artifacts.

In the research community of human-computer interaction, the experiences associated with the use of artifacts are discussed under the rubric of user experience (UX). While multiple UX definitions have been proposed, they share a focus on the experiences associated with artifact use. For example, Roto et al. [18, p. 6] state that "UX is a subset of experience as a general concept. UX is more specific, since it is related to the experiences of using a system." Some definitions restrict UX to actual system use [3], others include anticipated use [13], and still others also include aesthetics [10]. These differences appear, however, minor compared to the shared focus on the experiences associated with artifact use. Well-documented experiences with computer artifacts in work settings include burnout, deskilling, frustration, and helplessness [e.g., 7, 16]. Countering such negative experiences is central to employee 
wellbeing. Replacing them with positive experiences would be an even nobler design goal.

In one of the relatively few studies of UX in a work context, Meneweger et al. [17] show that ordinary user experiences dominate in factory employees' interactions with technology. The interactions are generally mundane, unremarkable, and shaped by routine activities. At the same time, studies of technology acceptance find that perceived enjoyment, a concept similar to UX, predicts the intention to use an artifact as strongly as do perceived usefulness and perceived ease of use [12]. While enjoyment contributes to hedonic wellbeing, usefulness contributes to eudaimonic wellbeing; ease of use facilitates both hedonic and eudaimonic wellbeing by reducing the effort that must be expended to obtain them. Thus, replacing ordinary user experiences with positive user experiences stands to improve employees' attitude toward the artifacts they use as well as to improve their wellbeing at work. The relation between the artifact and the user's experience is, however, complex because UX is not determined by the artifact alone. Rather, UX results from the interrelations among the characteristics of the artifact, user, task, and context of use.

While it is a largely trivial observation that UX results from the interrelations among the artifact, user, task, and context of use, it raises the question of whether artifacts as such exert much influence on wellbeing at work. It may well be that wellbeing at work is first and foremost driven by other factors, such as the task content, division of labor, physical work conditions, psychosocial climate, and decision-making influence. These factors are not directly about artifacts and, thereby, not directly about UX. To explore what we might accomplish by designing for good UX at work this study revisits Kammersgaard's [15] four perspectives on humancomputer interaction, ponders what constitutes positive and negative UX within each perspective, and discusses the possible contributions of UX to wellbeing at work.

\section{Four Perspectives on System Use and UX}

Kammersgaard [15] outlines four perspectives on human-computer interaction by distinguishing between artifacts for individual and collaborative use and between artifacts for which agency rests with the user and artifacts that split agency between user and artifact. The four perspectives are the tool perspective, the system perspective, the dialogue-partner perspective, and the media perspective, see Table 1.

The tool perspective has its roots in craftwork and emphasizes that in the hands of a skilled user the tool is a seamless extension of the user, who attends to her task rather than to the tool: When hammering the skilled user's attention is on driving the nail, not on the hammer. Conceptually, the tool is said to be ready to hand [9]. It is only upon breakdowns that the tool becomes the focus of the user's attention becomes present at hand. If the hammer is too light for the size of nail or otherwise inadequate for the task then the user's attention shifts from the task to the tool. These shifts are associated with frustration and other negative emotions because the breakdown thwarts progress on the task, at least temporarily. It appears that tools foster good experiences when they are out of mind - ready to hand - and poor 
experiences when they become present at hand. If we take the focus on artifact use in the UX definitions to mean that the user must, in the moment, be conscious that she is using an artifact then the tool perspective rules out positive UX. The positive experiences do not qualify as UX because they are associated with an uninterrupted focus on the task (note that this point will be modified in Section 3). In contrast, the user is conscious of the artifact when it thwarts task progress; thus tools can foster negative UX. If we do not require that the user must, in the moment, be conscious that she is using an artifact - and this is probably the more sensible option - then positive UX is possible within the tool perspective and consists of designing for readiness to hand. The user may however not attribute the positive UX to the tool but, partly or wholly, to other aspects of the use situation.

Table 1. Four perspectives on system use, adapted from Kammersgaard [15].

\begin{tabular}{lll}
\hline & Individual & Collaborative \\
\hline User agency & Tool perspective & Media perspective \\
& - Artifact is an extension of the user's & - Users communicate through the \\
body & artifact \\
& - Ready to hand vs present at hand & - Media richness vs common ground \\
- UX?: expressive power & - UX?: transparency, structure \\
Split agency & Dialogue-partner perspective & System perspective \\
& - Artifact displays human-like & - User is similar to other system \\
behavior & components \\
- Intelligent vs annoying assistant & - Automation vs meaningful jobs \\
- UX?: perceptive, adaptive & - UX?: deskilling, monotony \\
\hline
\end{tabular}

The system perspective aligns with industrial perceptions of work and promotes a view in which a system consists of components that may be human or automated. Each component is characterized by the input it receives, the activities it performs on those inputs, and the outputs it delivers. The division of the system into components is made by management and defines a division of labor. To perform their work the users need only know the characteristics of the component they embody. Performance is measured by how cheaply, quickly, and consistently the components deliver their outputs. That is, the users' work is measured in the same way as that of the automated components. If the users perform poorer than an automated version of the same component then the users are at risk of being replaced by such an automated component. In this sense the users are measured by their ability to function as automated components. The automation inherent in the system perspective is often associated with deskilling of the users, who become operators of machines that perform more and more components of the work [1]. This negative UX results from a primary focus on automation, thereby leaving the users with the components that have not yet been automated. To create positive UX it is necessary to focus on creating meaningful and rewarding human components, for example by automating the parts of work that are monotonous or unhealthy. However, to create meaningful and rewarding human components it may also be necessary to reconsider the separation between a managerial level that defines the components and an operational level that 
merely performs according to these preset definitions. That is, it may be necessary to challenge the essence of the system perspective.

The dialogue-partner perspective sees the artifact as an intelligent assistant with which the users can interact in much the same way as they interact with humans. The intelligent assistant empowers the user by serving his or her needs and does so without requiring that the user learns special commands for interacting with the assistant. Unlike the system perspective, which tends to reduce humans to machines, the dialogue-partner perspective seeks to elevate machines to human-like performance. Unlike the tool perspective, which involves the user's moment-tomoment handling of the tool, the intelligent assistant acts autonomously in the user's service. The intelligent assistant may, for example, monitor an architect's work on a building and inform the architect when his current building design violates formal regulations or recognized principles for good design [5]. The intelligent assistant fosters positive and negative UX in much the same way as a human collaborator. Negative UX ensues if the assistant needs too many instructions, performs poor work, or delivers its work at inopportune moments. Positive UX ensues if the assistant is effective and efficient and, especially, if the assistant also picks up on the tacit conditions for good performance and reacts appropriately to dynamic changes in the environment. Often, intelligent assistants must be supervised by users who need to be ready to take over in situations the assistant cannot handle. This creates poor conditions for positive UX because the user wants to offload the task to the assistant but must, instead, "stay in the loop" to be ready to step in whenever needed.

The media perspective positions the artifact as a medium through which the users interact with each other. That is, the medium is merely a channel; agency rests with the users. Rich media [19] provide for simultaneous interactions in multiple modalities and, thereby, for back-channeling (e.g., nods and raised eyebrows) to occur via some modalities at the same time as the main interaction occupies other modalities (e.g., speech). Thereby, rich media support users in establishing, sustaining, and repairing common ground, which is key to effective collaborative interactions. Conversely, lean media provide few or only a single modality and may be restricted to asynchronous interactions, thereby increasing the risk of breakdowns in common ground. Media provide positive UX when they are transparent - somewhat similar to when a tool is ready to hand. A transparent medium allows the interactions among the users to flow without distortions. Rich media are transparent with respect to more interaction modalities than lean media. In addition to transparency, some media aim to provide positive UX by structuring the interaction, for example by making explicit that an interactional turn is a request and therefore must be answered by accepting, declining or negotiating the request [21]. Media foster negative UX when they are insufficiently transparent or enforce a structure that is too rigid. In both cases the medium gets in the way of the interactions among the users. 


\section{Discussion}

Most work involves the use of artifacts, such as products, systems or services. Thus, UX is a factor in how most employees experience their work. In the tool and media perspectives, an artifact fosters positive UX by not attracting the user's attention, which instead remains on the task. That is, it is by supporting the user in expressing her skills - as manifested in high-quality work task products - that tools and media foster positive UX. Seen from these perspectives positive UX is about lending the user expressive power. In the dialogue-partner perspective, positive UX is as much about how well the artifact engages in the process of its use as it is about the product that results from this process. That is, an artifact fosters positive UX if it is a perceptive and adaptive dialogue partner. In the system perspective, positive UX appears to be secondary to other concerns. That is, to foster positive UX it is necessary to abandon the system perspective or, at least, supplement it with other perspectives. Abandoning the system perspective is a daunting undertaking because this perspective permeates much thinking about how to organize workplaces. For example, physicians are increasingly frustrated that they spend still more of their time documenting their work in electronic patient records and comparatively less time with patients, but the increasing documentation requirements are justified by pointing out that the physician is a component in a much larger system, which needs the documentation for hospital-level quality assurance, national performance indicators, and international healthcare research [7]. This system-perspective thinking is, however, creating frustration and burnout among the physicians because it disregards the personal level from which they experience the electronic patient records.

A less ambitious goal than fostering positive UX in the service of wellbeing at work would be to avoid negative UX. The tool and media perspectives agree that artifacts foster negative UX whenever they attract the user's attention. Thus, users become conscious of their artifact use when they experience problems with the artifacts. The distinction between, on the one hand, positive UX and a task focus and, on the other hand, negative UX and an artifact focus largely stems from the distinction between the concepts of ready to hand and present at hand. Recently, Verbeek [20] has proposed that artifacts need not be either ready to hand or present at hand, but can be both at the same time. He illustrates this possibility by considering the difference between a CD player and a piano [20, p. 194]:

Someone who plays the piano is directed toward the music and at the same time is substantially involved with the piano itself. When the same piece is played on a CD player, the artifact that mediates between the person and the music is present in an entirely different way. The machinery of a CD player disappears into the background, withdrawing so that people are only engaged with the music and not with its means of production. A piano, however, is never entirely ready-to-hand, but neither is it exclusively present-at-hand-its machinery is not completely in the background, but not entirely in the foreground either.

In this example the CD player functions as the perfect assistant, to which the task of playing the music can be completely offloaded. In contrast, the piano requires that the 
human stays in the loop and operates the piano on a moment-to-moment basis. By proposing that pure readiness to hand is only achieved with complete offloading (i.e., with full automation), Verbeek [20] proposes that pure readiness to hand implies that the human is out of the loop. This proposal fundamentally reconceptualizes readiness to hand by dissociating it from skilled human performance. According to Verbeek [20], skilled human performance instead involves that the artifact is simultaneously ready to hand and present at hand - like a piano or a hammer.

Verbeek [20] also contends that a purely present-to-hand artifact does not necessarily indicate a negatively experienced breakdown in the use of the artifact; it may also indicate that the user is absorbed in cherishing the artifact rather than in using it as a means to an end. This contention accords with many UX studies of users' experiences of the aesthetic and other non-instrumental qualities of their possessions [e.g., 8, 14]. Cherishing may to some extent be about possessing the artifact and, thereby, not fully applicable to artifacts that are operated by the user but owned by the workplace. Yet, a sizable number of people have work tools available in a near permanent manner that approaches ownership.

\section{Conclusion}

Verbeek's [20] elaboration of the concepts of ready to hand and present at hand offers a way of restructuring and simplifying the insights from the analysis of the four perspectives on how UX may contribute to wellbeing at work, see Table 2. This restructuring leads to three conclusions:

First, when artifacts are present at hand the users focus on the artifact rather than their tasks. While this artifact focus may be associated with positive, artifactcherishing experiences, it is often triggered by breakdowns in artifact use. To contribute to wellbeing at work artifacts must be designed so that it is easy to restore their functioning after a breakdown.

Second, when artifacts are simultaneously present at hand and ready to hand the users are conscious that they are using an artifact but their focus is on their task. Following the tool and media perspectives, artifacts that enable a task focus foster positive UX by lending the users expressive power, by being transparent and, possibly, by structuring the interaction. Importantly, Verbeek's [20] reconceptualization does away with the question of whether positive UX is possible within the tool perspective.

Table 2. What may UX contribute to wellbeing at work?

\begin{tabular}{|c|c|c|}
\hline Artifact is... & Focus & UX contribution to wellbeing at work \\
\hline Present at hand & Artifact & $\begin{array}{l}\text { - Breakdown in use (negative UX) } \\
\text { - Cherishing the artifact (positive UX) }\end{array}$ \\
\hline $\begin{array}{l}\text { Both present at hand } \\
\text { and ready to hand }\end{array}$ & Task & $\begin{array}{l}\text { Expressive power, transparency, and possibly } \\
\text { structure (positive UX) }\end{array}$ \\
\hline Ready to hand & Consumption & $\begin{array}{l}\text { - Perceptive and adaptive assistant (positive UX) } \\
\text { - Deskilling and monotony (negative UX) }\end{array}$ \\
\hline
\end{tabular}


Third, when artifacts are ready to hand they autonomously produce outputs for the users to consume. This consumption focus is experienced positively when the artifact serves the user as a perceptive and adaptive assistant and negatively when the user must abide the system. Thus, the dialogue-partner perspective may foster positive UX through ready-to-hand artifacts, while the system perspective fosters negative UX by leaving the user out of not just the activity loop but also the decision loop.

It should be noted, in closing, that the four perspectives revisited in this study are exclusively about post-design experiences with artifacts. Neither the four perspectives, nor the concepts of ready to hand and present at hand, concern themselves with how artifacts are designed. Yet, user influence on the design of the artifacts employed in performing work tasks may be an additional UX contribution to wellbeing at work [11].

\section{References}

1. Braverman, H.: Labor and monopoly capital: The degradation of work in the twentieth century. Monthly Review Press, New York (1974).

2. Chen, P.Y., Cooper, C. (Eds.). Wellbeing: A complete reference guide. Volume III: Work and wellbeing. Wiley, New York (2014).

3. Colbert, M.: User experience of communication before and during rendezvous: Interim results. Personal and Ubiquitous Computing 9(3), 134-141 (2005).

4. Cooper, C.L., Leiter, M.P. (Eds.). The Routledge companion to wellbeing at work. Routledge, New York (2017).

5. Fischer, G., Lemke, A.C., Mastaglio, T.: Critics: An emerging approach to knowledgebased human-computer interaction. International Journal of Man-Machine Studies 35(5), 695-721 (1991).

6. Fisher, C.D.: Conceptualizing and measuring wellbeing at work. In: Chen, P.Y., Cooper, C.L. (eds.) Wellbeing: A Complete Reference Guide. Volume III: Work and Wellbeing, pp. 9-33. Wiley, New York (2014).

7. Gawande, A.: The upgrade: Why doctors hate their computers. The New Yorker, 62-73 (November 12, 2018).

8. Hassenzahl, M., Tractinsky, N.: User experience - A research agenda. Behaviour \& Information Technology 25(2), 91-97 (2006).

9. Heidegger, M.: Being and time (Macquarrie, J., Robinson, E., Trans.). Harper, New York (1962/1927).

10. Hekkert, P.: Design aesthetics: Principles of pleasure in product design. Psychology Science 48(2), 157-172 (2006).

11. Hertzum, M., Torkilsheyggi, A.: How do users perceive a design-in-use approach to implementation? A healthcare case. In: Proceedings of the INTERACT2019 Conference on Human-Computer Interaction, Vol. LNCS 11748, pp. 410-430. Springer, Cham (2019).

12. Hornbæk, K., Hertzum, M.: Technology acceptance and user experience: A review of the experiential component in HCI. ACM Transactions on Computer-Human Interaction 24(5), Article 33 (2017). 
13. ISO 9241: Ergonomics of human-system interaction - Part 210: Human-centred design for interactive systems. International Standard Organization, Geneva, CH (2010).

14. Jordan, P.W.: Human factors for pleasure in product use. Applied Ergonomics 29(1), 25-33 (1998).

15. Kammersgaard, J.: Four different perspectives on human-computer interaction. International Journal of Man-Machine Studies 28(4), 343-362 (1988).

16. Lazar, J., Jones, A., Shneiderman, B.: Workplace user frustration with computers: An exploratory investigation of the causes and severity. Behaviour \& Information Technology 25(3), 239-251 (2006).

17. Meneweger, T., Wurhofer, D., Fuchsberger, V., Tscheligi, M.: Factory workers' ordinary user experiences: An overlooked perspective. Human Technology 14(2), 209-232 (2018).

18. Roto, V., Law, E., Vermeeren, A., Hoonhout, J.: User experience white paper: Bringing clarity to the concept of user experience. Result from Dagstuhl Seminar (2011). http://www.allaboutux.org/uxwhitepaper (accessed April 1, 2019).

19. Trevino, L.K., Lengel, R.H., Daft, R.L.: Media symbolism, media richness, and media choice in organizations: A symbolic interactionist perspective. Communication Research 14(5), 553-574 (1987).

20. Verbeek, P.-P.: What things do: Philosophical reflections on technology, agency, and design. Pennsylvania State Unversity Press, University Park, PA (2005).

21. Winograd, T., Flores, F.: Understanding computers and cognition: A new foundation for design. Ablex, Norwood, NJ (1986). 